ИЗВЕСТИЯ АКАДЕМИИ НАУК ЭСТОНСКОИ ССР. ФИЗИКА МАТЕМАТИКА

PROCEEDINGS OF THE ACADEMY OF SCIENCES OF THE ESTONIAN SSR. PHYSICS * MATHEMATICS

$1988,37,3$

УДК 537.226.4

П. КОНСНН, Т. ЭРД

\title{
СЕГНЕТОЭЛЕКТРИЧЕСКИЕ ФАЗОВЫЕ ПЕРЕХОДЫ ПЕРВОГО РОДА ВО ВНЕШНЕМ МАГНИТНОМ ПОЛЕ
}

\author{
(Представил В. Хижняков)
}

Исследовано влияние магнитного поля на сегнетоэлектрические фазовые переходы типа смещения в системах с сильной вибронной ангармоничностью. Показано, что магнитное поле может вызвать изменение рода фазового перехода. Рассмотрены переходы первого рода в магнитном поле. Предсказан температурный магнитогистерезисный эффект.

Настоящая работа посвящена исследованию сегнетоэлектрических фазовых переходов типа смещения при наличии внешнего магнитного поля $B$ в кристаллах с существенной вибронной ангармоничностью. Это означает, что в разложении Ландау части свободной энергии системы, связанной с межзонным электрон-фононным взаимодействием, учитываются члены более высокого порядка, чем квадратичные. Ранее на основе вибронной теории структурных фазовых переходов (см. напр., $\left.\left[{ }^{1-5}\right]\right)$ в основном изучалась зависимость частоты мягкой моды от $B\left[{ }^{6-14}\right]$.

Разложение свободной энергии вибронного сегнетоэлектрика в магнитном поле $\left[{ }^{8}\right]$ по степеням фононного параметра порядка $y_{0}$ имеет вид

$$
\begin{gathered}
F\left(y_{0}\right)=F(0)+\frac{M}{2} \Omega_{h s}^{2}(T, B) y_{0}^{2}+\frac{1}{2 N_{0}}\left[u_{1}+v_{1}(T, B)\right] y_{0}^{4}+ \\
+\frac{1}{6 N_{0}^{2}}\left[u_{2}+v_{2}(T, B)\right] y_{0}^{6}
\end{gathered}
$$

Здесь $\Omega_{h s}$ и $v_{1,2}$ - частота мягкой моды в парафазе и коэффициенты вибронной ангармоничности 4-го и 6-го порядка соответственно, $u_{1,2}$ - коэффициенты собственной фононной ангармоничности, $M$ - приведенная масса, отвечающая сегнетоактивному колебанию, $N_{0}-$ число элементарных ячеек. Предполагаем, что валентная зона заполнена $N$ электронами, а зона проводимости - пустая. Используя для электронных $3 о н$ изотропные параболические спектры $\left(\left|\delta_{n, p}\right|, \hbar \omega_{c}{ }^{n, p} \ll \Delta\right.$; $\hbar \omega_{c}{ }^{n, p} \ll E_{0}(0) ; E_{0}(0)$ - суммарная ширина разрешенных $30 н$ при $B=0, \Delta-$ затравочная ширина запрещенной зоны, $\delta_{n, p}$ и $\omega_{c}^{n, p}-$ спиновые расщепления и циклотронные частоты электронов и дырок), получается

$$
\begin{gathered}
M \Omega_{h s}^{2}(T, B)=M \hat{\omega}_{0}^{2}(T)-2 \bar{V}^{2}[\bar{\Delta}(B)]^{-1}, \\
{[\bar{\Delta}(B)]^{-1}=[\bar{\Delta}(0)]^{-1}+\frac{3 x_{3}(B)}{4 E_{0}^{3 / 2}(0)}}
\end{gathered}
$$




$$
\begin{gathered}
{[\bar{\Delta}(0)]^{-1}=\frac{3}{E_{0}(0)}\left[1-\sqrt{\frac{\Delta}{E_{0}(0)}} \operatorname{arctg} \sqrt{\frac{E_{0}(0)}{\Delta}}\right], \quad \bar{V}=V \sqrt{\frac{N}{N_{0}}}} \\
v_{1}(B)=v_{1}(0)+\frac{3 \bar{V}^{2} V^{2} x_{5}(B)}{2 E_{0}^{3 / 2}(0)}, \\
v_{1}(0)=\frac{3 \bar{V}^{2} V^{2}}{2 E_{0}^{3 / 2}(0)} J_{1}(3) \\
v_{2}(B)=v_{2}(0)-\frac{9 \bar{V}^{2} V^{4} x_{7}(B)}{E_{0}^{3 / 2}(0)} \\
v_{2}(0)=-\frac{3 \bar{V}^{2} V^{4}}{2 E_{0}^{3 / 2}(0)} J_{1}(5) .
\end{gathered}
$$

Здесь

$$
\begin{gathered}
x_{k}(B)=\left(\delta_{p}-\delta_{n}\right)^{2} J_{1}(k)-\frac{\left(\hbar \overline{\omega_{c}}\right)^{2}}{3} J_{2}(k-1) \\
J_{1}(k)=-\frac{\sqrt{E_{0}(0)}(k-2)}{\left(E_{0}(0)+\Delta\right)^{k-1}+J_{2}(k-1), \quad k \geqslant 3} \\
J_{2}(k)=\frac{(2 k-3) ! !}{(k-2) ! 2}\left\{\sqrt{E_{0}(0)} \sum_{l=1}^{k-1} \frac{(k-1-l) !}{(2 k-1-2 l) ! ! \Delta^{l}\left(E_{0}(0)+\Delta\right)^{k-l}}+\right. \\
\left.+\frac{1}{2^{k-2} \Delta^{k-1 / 2}} \operatorname{arctg} \sqrt{\frac{E_{0}(0)}{\Delta}}\right\}, \quad k \geqslant 2 \\
\omega_{c}^{n, p}=\frac{e B}{m_{n, p}}, \quad \delta_{n, p}=\frac{1}{2} g_{n, p} \mu_{B} B \\
\bar{\omega}_{c}=\frac{e B}{\bar{m}}, \quad \frac{1}{\bar{m}}=\frac{1}{2}\left(\frac{1}{m_{n}}+\frac{1}{m_{p}}\right)
\end{gathered}
$$

$m_{n, p}$ и $g_{n, p}-$ эффективные массы и факторы спинового расщепления электронов и дырок, $V$ - константа межзонного электрон-фононного взаимодействия, $\hat{\omega}_{0}(T)-$ перенормированная фонон-фононными взаимодействиями затравочная частота сегнетоэлектрического колебания $[1,3,5]$. При $E_{0} \gg \Delta$

$$
J_{1}(k) \approx J_{2}(k-1) \approx J_{0}(k),
$$

причем

$$
J_{0}(k)=\frac{\pi(2 k-5) ! !}{2^{k-1} \Delta^{k-3 / 2}(k-3) !}, \quad k \geqslant 3
$$

и

$$
\chi_{k}(B) \approx\left[\left(\delta_{p}-\delta_{n}\right)^{2}-\frac{\left(\hbar \bar{\omega}_{c}\right)^{2}}{3}\right] J_{0}(k) .
$$

В связи со свободной энергией (1) должно выполняться условие устойчивости системы $u_{2}+v_{2}(B)>0$, откуда с учетом (7) получаем

$$
x_{7}(B)<\frac{E_{0}^{3 / 2}(0)\left[u_{2}+v_{2}(0)\right]}{9 \bar{V}^{2} V^{4}} .
$$


В случае $x_{7}(B)>0$ условие (17) ограничивает сверху индукцию приложенного поля. Если $\varkappa_{7}(B)<0$, то $(17)$ выполняется всегда.

Зависимость коэффициента вибронной ангармоничности 4-го . порядка $v_{1}$ от $B$ приводит к возможности изменения рода структурного фазового перехода во внешнем магнитном поле. Если при $B=0$ происходит переход первого рода $\left(u_{1}+v_{1}(0)<0, u_{2}+v_{2}(0)>0\right)$, то при

$$
\lambda>\sqrt{\frac{J_{2}(4)}{3 J_{1}(5)}}, \quad B>B_{\mathrm{cr}},
$$

коэффициент $u_{1}+v_{1}(B)$ становится положительным и происходит фазовый переход второго рода. В (18)

$$
\begin{gathered}
\lambda=\frac{\left|g_{p}-g_{n}\right| \bar{m}}{2 m_{0}}, \\
B_{c r}=\left[\bar{V} V \mu_{B}\right]^{-1}\left\{2 E_{0}^{3 / 2}(0)\left|u_{1}+v_{1}(0)\right| \times\right. \\
\left.\times\left|\frac{3}{4}\left(g_{p}-g_{n}\right)^{2} J_{1}(5)-\left(\frac{2 m_{0}}{\bar{m}}\right)^{2} J_{2}(4)\right|^{-1}\right\}^{1 / 2},
\end{gathered}
$$

$m_{0}$ - масса свободного электрона. Из анализа условий (17), (18) с учетом (19), (20) следует возможность их одновременного выполнения.

Превращение фазового перехода второго рода $\left(u_{1}+v_{1}(0)>0\right.$, $\left.u_{2}+v_{2}(0)>0\right)$ в переход первого рода имеет место при

$$
\lambda<\sqrt{\frac{J_{2}(4)}{3 J_{1}(5)}}, \quad B>B_{\mathrm{cr}} .
$$

Следовательно, спиновое расщепление с $g_{p} \neq g_{n}$ благоприятствует сегнетоэлектрическому фазовому переходу второго рода, а циклотронное квантование - фазовому переходу первого рода.

Рассмотрим далее влияние магнитного поля на структурные фазовые переходы первого рода $\left(u_{1}+v_{1}(B)<0, u_{2}+v_{2}(B)>0\right)$. Из условия минимума свободной энергии (1) с учетом (2)-(8) находим спонтанное искажение решетки $y_{00}$

$$
\begin{gathered}
y_{00}^{2}(T, B) / N_{0}=\left\{-\left[u_{1}+v_{1}(B)\right]+\left\{\left[u_{1}+v_{1}(B)\right]^{2}-\right.\right. \\
\left.\left.-\left[u_{2}+v_{2}(B)\right] M \Omega_{h s}^{2}(T, B)\right\}^{1 / 2}\right\}\left[u_{2}+v_{2}(B)\right]^{-1}
\end{gathered}
$$

Температура фазового перехода первого рода в магнитном поле $T_{0}(B)$ определяется из условия равновесия поляризованной и неполяризованной фаз $F\left(T_{0}(B), y_{00}\left(T_{0}(B), B\right)\right)=F\left(T_{0}(B), 0\right)$, откуда при $\left|\Delta T_{c}(B)\right| \ll T_{c}(0) ; \quad\left|\Delta T_{0}(B)\right| \ll T_{0}(0) \quad$ (а также в высокотемпературном пределе $\left.\left[{ }^{1}\right]\right)$ получаем

$$
\begin{gathered}
\Delta T_{0}(B)=\Delta T_{c}(B) \sigma_{0}+\frac{3}{4 k_{B} \gamma\left(T_{0}(0)\right)}\left\{\frac{\left[u_{1}+v_{1}(B)\right]^{2}}{u_{2}+v_{2}(B)}-\frac{\left[u_{1}+v_{1}(0)\right]^{2}}{u_{2}+v_{2}(0)}\right\} \\
\sigma_{0}=\gamma\left(T_{c}(0)\right) / \gamma\left(T_{0}(0)\right) .
\end{gathered}
$$

Здесь $\Delta T_{0}(B)=T_{0}(B)-T_{0}(0) ; \Delta T_{c}(B)=T_{c}(B)-T_{c}(0), T_{c}$ - температура, при которой $\Omega_{h s}$ обращается в нуль; $\quad \gamma(T)=\partial \hat{\hat{\omega}_{0}^{2}}(T) / \partial k_{B} T$; коэффициенты вибронного ангармонизма $v_{1,2}(B)$ даны формулами (5), $(7)$, а $\Delta T_{c}(B)$ согласно $(2),(3)$ равен 


$$
\Delta T_{c}(B)=\frac{3 \bar{V}^{2} \varkappa_{3}(B)}{2 E_{0}^{j_{2}}(0) k_{B} \gamma\left(T_{c}(0)\right)} .
$$

Если магнитные сдвиги $v_{1,2}$ достаточно малы, т. е. $\left|v_{1}(B)-v_{1}(0)\right| \ll$ $\ll\left|u_{1}+v_{1}(0)\right| ;\left|v_{2}(B)-v_{2}(0)\right| \ll u_{2}+v_{2}(0)$, то с учетом (5), (7) можно (23) приближенно представить в виде

$$
\begin{aligned}
\Delta T_{0}(B)= & \Delta T_{c}(B) \sigma_{0}+\frac{9 \bar{V}^{2} V^{2}\left(u_{1}+v_{1}(0)\right)}{4 k_{B} \gamma\left(T_{0}(0)\right) E_{0}^{j_{2}}(0)\left(u_{2}+v_{2}(0)\right)} \times \\
& \times\left\{\varkappa_{5}(B)+\frac{3 V^{2}\left(u_{1}+v_{1}(0)\right)}{u_{2}+v_{2}(0)} \varkappa_{7}(B)\right\} .
\end{aligned}
$$

При $E_{0} \gg \Delta$ на основе $(25)$, (26), (16) получаем для отношения магнитных сдвигов $T_{0}$ и $T_{c}: S_{0} \equiv \Delta T_{0}(B) / \Delta T_{c}(B)$

$$
S_{0}=\sigma_{0}\left(1-\frac{45}{16} \theta+\frac{2835}{256} \theta^{2}\right) \text {, }
$$

где

$$
\theta=\frac{V^{2}\left|u_{1}+v_{1}(0)\right|}{\Delta^{2}\left(u_{2}+v_{2}(0)\right)}
$$

Из (27) следует, что направления магнитных сдвигов $T_{0}$ и $T_{c}$ совпадают, так как правая сторона формулы (27) всегда положительна. При этом *

$$
\begin{gathered}
S_{0} \gtrless 1, \text { если } \theta \gtrless \theta_{0}, \\
\theta_{0}=\frac{8}{63}\left[1+\sqrt{1+\frac{28}{5}\left(\sigma_{0}^{-1}-1\right)}\right] .
\end{gathered}
$$

Отметим также, что функция $S_{0}(\Theta)$ проходит через минимум при $\theta=\frac{8}{63}<\theta_{0}$. Зависимость $S_{0}$ от $\Theta$ для $\sigma_{0}=1$ приведена на рисунке.

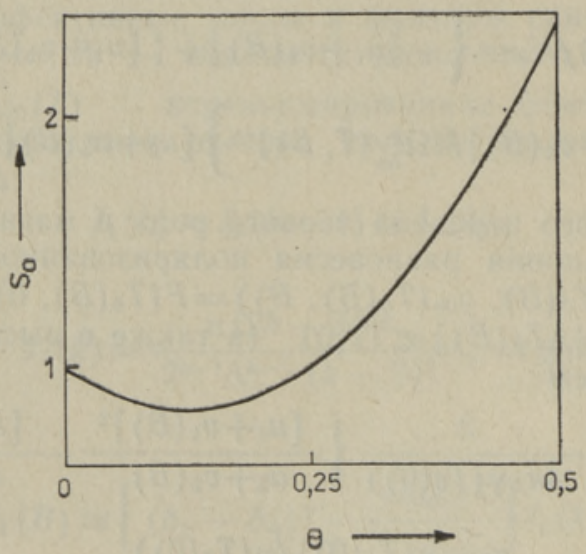

Зависимость $S_{0}$ от $\Theta$ при $\sigma_{0}=1$.

* Предполагается, что $\gamma(T)$ - монотонно возрастающая функция. В высокотемпературном пределе $\gamma(T)=$ const. Следовательно, $\sigma_{0} \leqslant 1$. 
Таким образом, магнитные сдвиги $\Delta T_{0}(B)$ и $\Delta T_{c}(B)$ в общем случае не равны. Росту сдвига $\Delta T_{0}(B)$ в сравнении с $\Delta T_{c}(B)$ благоприятствуют близость $\sigma_{0}$ к единице и достаточно большое значение параметра $\Theta$. Величины смещений $\Delta T_{c}(B)$ оценены в $[8,11,13,15]$.

Формула (27) применима в случае относительно слабого магнитного поля, когда

$\left|\Delta T_{0}(B)-\sigma_{0} \Delta T_{c}(B)\right| \ll \frac{M}{k_{B} \gamma\left(T_{0}(0)\right)}\left[\hat{\omega}_{0}^{2}\left(T_{0}(0)\right)-\hat{\omega}_{0}^{2}\left(T_{c}(0)\right)\right] . \quad$ Для более сильных полей справедливо $(23)$, причем отношение $S_{0}$ зависит тетерь от индукции $B$.

Когда сегнетоэлектрик типа смещения испытывает фазовый переход первого рода, можно ожидать влияния внешнего магнитного поля на температурный гистерезис перехода (магнитогистерезисный эффект). Максимальная ширина температурного гистерезиса фазового перехода первого рода определена следующим образом

$$
\Delta T_{h}(B)=T_{1}(B)-T_{c}(B),
$$

где $T_{1}$ - температура, выше которой существует только парафаза**. Перепишем (31) в виде

$$
\Delta T_{h}(B)=\Delta T_{h}(0)+\alpha_{h}(B),
$$

где величина изменения максимальной ширины температурного гистерезиса

$$
\alpha_{h}(B)=\Delta T_{1}(B)-\Delta T_{c}(B) .
$$

Аналогично формуле (27) можно получить выражение для огношения $S_{1} \equiv \Delta T_{1}(B) / \Delta T_{c}(B)$

$$
\begin{gathered}
S_{1}=\sigma_{1}\left(1-\frac{15}{4} \theta+\frac{945}{64} \theta^{2}\right), \\
\sigma_{1}=\gamma\left(T_{\mathrm{c}}(0)\right) / \gamma\left(T_{1}(0)\right) .
\end{gathered}
$$

Из (34) следует, что обе границы области бифуркации фаз смещаются в магнитном поле одновременно в сторону более высоких или низких температур (см. также $\left[{ }^{15}\right]$ ) в зависимости от значения параметра $\lambda$. Знак $\alpha_{h}(B)$ определяется условиями

$$
\begin{array}{ll}
\lambda>1 / \sqrt{3}: \alpha_{h}(B) \gtrless 0, \text { если } \theta \gtrless \theta_{1}, \\
\lambda<1 / \sqrt{3}: \alpha_{h}(B) \lessgtr 0, \text { если } \theta \gtrless \theta_{1},
\end{array}
$$

где

$$
\theta_{1}=\frac{8}{63}\left[1+\sqrt{1+\frac{21}{5}\left(\sigma_{1}^{-1}-1\right)}\right]
$$

Таким образом, изучены особенности структурных фазовых переходов первого рода во внешнем магнитном поле. В частности, предсказан температурный магнитогистерезисный эффект в сегнетоэлектриках типа смещения.

Авторы благодарны Н. Кристофелю за обсуждение результатов работы.

\footnotetext{
** В условиях температурного гистерезиса переход первого рода из сегнетофазы в парафазу происходит при некоторой температуре $T^{\prime}: T_{0} \leqslant T^{\prime} \leqslant T_{1}$, а из парафазы в сегнетофазу при температуре $T^{\prime \prime}: T_{0} \geqslant T^{\prime \prime} \geqslant T_{\mathrm{c}}$.
} 
1. Kristoffel, N., Konsin, P. Ferroelectrics, 6, 3-12 (1973).

2. Konsin, P. Phys. status solidi (b), 76, № 2, 487-496 (1976).

3. Konsin, P. Phys, status solidi (b), 86, Ng 1, 57-66 (1978).

4. Bersuker, I. B., Vekhter. B. G. Ferroelectrics, 19, 137-150 (1978).

5. Kristoffel, N. N., Konsin, P. J. Ferroelectrics, 21, № 1-4, 477-479 (1978).

6. Вехтер Б. Г., Зенченко В. П., Берсукер И. Б. ФТТ, 18, вып. 8, 2325-2330 (1976).

7. Takaoka, S., Murase, K. Phys. Rev. B, 20, № 7, 2823-2833 (1979).

8. Konsin, P., Ord, T. Phys. status solidi (b), 97, № 2, 609-615 (1980).

9. Волков В. Л., Литвинов В. И. ФТТ, 22, вып. 2, 617-619 (1980).

10. Volkov, V. L., Litvinov, V. I. Phys. Lett. (A) 75, № 5, 398-400 (1980).

11. Konsin, P., Ord, T. Ferroelectrics, 45, $121-129$ (1982).

12. Зенченко В. П., Вехтер Б. Г., Берсукер И. Б. ЖЭТФ, 28, вып. 5, 1628-1639 (1982).

13. Konsin, P., Ord, T. In: Synergetics. Proc. Int. Symp. «Synergetics and Cooperative Phenomena in Solids and Macromolecules». Tallinn, «Valgus», 1983, $71-82$.

14. Konsin, P., Kristoffel, N., Ord, T. Solid State Commun., 49, № 4, 351-355 (1984).

15. Кристофель Н., Консин П., Эрд Т. Изв. АН ЭССР. Физ. Матем., 36, № 1, 8-13 (1987).

Ннститут физики

Академии наук Эстонской ССР

\section{Поступила в редакцию} 11/VI 1987

\section{P. KONSIN, T. ORD}

\section{ESIMEST LIIKI SENJETTELEKTRILISED FAASISIIRDED VÄLISES MAGNETVÄLJAS}

On uuritud magnetvälja mõju nihke tüüpi senjettelektrilistele faasisiiretele tugeva vibroonse anharmoonilisusega süsteemides ning näidatud, et magnetväli võib põhjustada faasisiirde liigi muutuse. On käsitletud esimest liiki faasisiirdeid magnetväljas ning ennustatud temperatuurilise magnethüstereesi efekti olemasolu.

\section{P. KONSIN and T. ORD}

\section{THE FIRST-ORDER FERROELECTRIC PHASE TRANSITIONS IN THE EXTERNAL MAGNETIC FIELD}

The influence of the magnetic field on displacive ferroelectric phase transitions in the systems with strong vibronic anharmonicity has been studied. It is shown that the magnetic field can cause the change of phase transition order. The first-order transitions in the magnetic field are considered. The temperature magnetohysteresis effect is predicted. 